\title{
MadWeight: automatic event reweighting with matrix elements
}

\author{
P. Artoisenet \\ Université Catholique de Louvain (UCL) \\ Center for Particle Physics and Phenomenology (CP3) \\ E-mail: pierre.artoisenet@uclouvain.be \\ O. Mattelaer* \\ Université Catholique de Louvain (UCL) \\ Center for Particle Physics and Phenomenology (CP3) \\ E-mail: olivier.mattelaer@uclouvain.be
}

\begin{abstract}
We present MadWeight, a new phase space generator aimed at efficiently computing the weights needed in the matrix element method. Given an arbitrary decay chain and a user-defined transfer function, the algorithm creates an optimized phase-space mapping to integrate the product of the square matrix element and the transfer function. We illustrate the code with some applications, such as the use of these weights to discriminate signal from background in charged Higgs production analyses.
\end{abstract}

Prospects for Charged Higgs Discovery at Colliders

September 16-19 2008

Uppsala, Sweden

${ }^{*}$ Speaker. 


\section{Introduction}

During the past few years, the top quark mass measurements performed by CDF and D0 collaborations have been improved significantly through the use of the matrix element method [1, 2, 3, 4]. In this approach, each experimental event is associated to a weight based on the square matrix element [5, 6]. The weights are then combined together into a likelihood to determine the most probable value of the top-quark mass. The method has also been used in other analyses, for example, in the search for the Standard Model Higgs produced in gluon fusion $g g \rightarrow H \rightarrow W W^{*}$ [何] or in the measurement of the single-top cross-section [8] at the Tevatron.

Although the method has become more and more popular, its practical application is not straightforward. The numerical computation of the weights requires to build a code specific to each process. In order to automate this step, we devised a new general algorithm to build automatically a phase-space mapping corresponding to a given amplitude that allows an efficient evaluation of the weights for a large class of processes.

In this talk, we will first present the definition of weights in the matrix element method. Secondly, we will explain why we need to build a specific phase-space generator for each process and how MadWeight succeeds to automate this step. Finally we will present two applications, the top-quark mass measurement, and the potential observation of a charged Higgs issues from the top quark decay.

\section{Matrix Element Method}

The matrix element method [9, 10] associates to each experimental event $[x]$ a weight $[P(x)]$ given by the probability to observe this event according to a given theoretical frame $[\alpha]$. The weight is defined as the convolution of the square matrix element with the resolution function $W$ (also named transfer function):

$$
P(x)=\frac{1}{\sigma} \int d \phi(y)\left|M_{\alpha}\right|^{2}(y) d w_{1} d w_{2} f_{1}\left(w_{1}\right) f_{2}\left(w_{2}\right) W(x, y),
$$

where $y$ is a parton-level phase-space point, $d \phi(y)$ is the phase-space measure and $f_{i}\left(w_{i}\right)$ are the Parton Distribution Functions. The normalization by the total cross section $\sigma$ in (2.1) ensures that $P(x)$ is a probability density: $\int P(x) d x=1$.

In principle, this definition provides the best discriminator on an event-by-event basis between different theoretical frames. In particular, the most probable value of $\alpha$ can be estimated through a likelihood maximization method [10].

\section{MadWeight}

Although this method is conceptually simple, the numerical evaluation of the weights $P(x)$ suffers from technical difficulties. The peaked behavior of the transfer function cannot be probed efficiently by the usual phase-space integrators and hence requires dedicated effort. 


\subsection{Adaptive Monte Carlo Integrator}

Monte Carlo (MC) integration is the most efficient technique to evaluate highly dimensional integrals. It consists in generating random points in order to estimate the integral. Even if this technique is very simple and general, it is very inefficient in presence of sharp peaks. The easiest way to improve the efficiency is to probe with more points the regions where the integrand is the largest in magnitude. This is achieved, for example, in an adaptive way by VEGAS [11]. This algorithm is only efficient for peaks aligned on the integration variables [12]. This means that a phase space parametrization has to be found where the integrand is of the form

$$
f(x)=\left(\prod_{i=1}^{n} P_{i}\left(\mathrm{x}_{i}\right)\right) \times F(x),
$$

where the functions $P_{i}$ 's may vary abruptly with the value of a single variable of integration $x_{i}$, while $F(x)$ is essentially flat all over the region under integration. If the integrand is of the form of Eq. (3.1) in the parameterization $x$, we say that all the peaks are factorized.

\subsection{MadWeight Integrator}

As we have seen in the previous section, the problem of evaluating the weights in Eq. (2.1) through a MC integration can be reduced to the problem of choosing an appropriate phase-space parameterization such that the sharp behavior of the integrand is factorized as in Eq. (3.1).

The abrupt part of the integrand in Eq. (2.1) is actually a product of one-dimensional functions. In the squared matrix element, the strong variations are caused by the propagators and are controlled by the invariant masses of the exchanged particles. On the other hand, the transfer function has a sharp dependence both in energy and in angles of any visible particle.

To obtain the appropriate parametrization of the phase-space, Madweight starts from the standard measure: $\prod_{i=1}^{N} \frac{d^{3} p_{i}}{(2 \pi)^{3} E_{i}}$ and then applies a series of analytical changes of variables. See [13] for more details.

\section{Examples of Applications}

In this section, we present some illustrations of the code. The following analyses are based on Monte Carlo events. This allows us to keep a control on the relevance of the results, and hence to validate our approach.

Unweighted events are generated with MadGraph/MadEvent [14]. These events are passed through Pythia [15] for the parton-shower and the hadronization. Finally the reconstruction in the detector is simulated by PGS [16], tuned to the CMS detector.

\subsection{Measurement of the top-quark mass}

The top quark-mass is an important quantity in the standard model, as its accurate value can be used to constrain the mass of not yet observed particles such as the Higgs. Several analyses based on the matrix element method have been used by CDF and D0 collaborations to measure $m_{t}$ from top-quark pair events (See [17] for a review). In this case the matrix element method provides a better accuracy on the mass than other methods like neural networks or kinematical fits. 


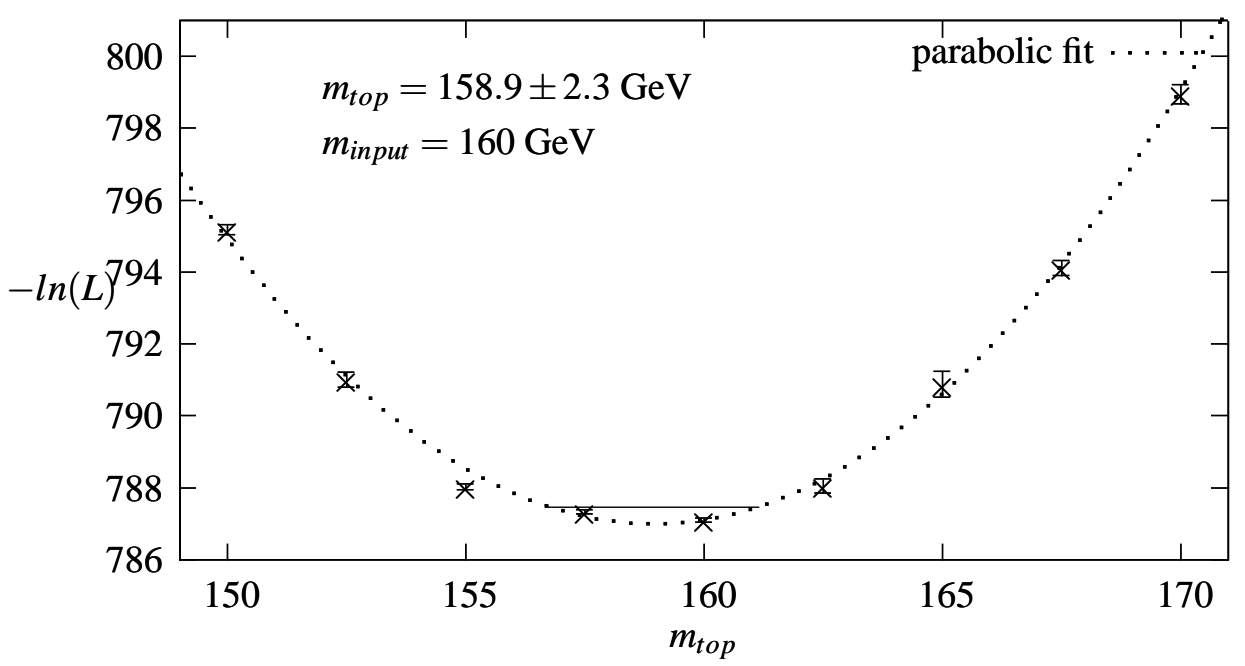

Figure 1: Plot of the likelihood for a sample of 20 events generated with $m_{t}$, input $=160 \mathrm{GeV}$. The value $m_{t}=158.9 \pm 2.3_{\text {stat }} \mathrm{GeV}$ has been extracted from the parabolic curve fitting the points.

In this first example, we consider the semi-leptonic channel of the top-quark pair production at the LHC collider:

$$
p p \rightarrow\left(t \rightarrow b\left(W^{+} \rightarrow j j\right)\right)\left(\bar{t} \rightarrow \bar{b}\left(W^{-} \rightarrow \mu^{-} \bar{v}_{\mu}\right)\right) .
$$

In first approximation we consider the transfer function associated to the jet energy parametrized by a double gaussian (we follow the parameterization introduced in [10]), all other quantities such as the direction of visible particles and the energy of leptons are well measured. In other words the corresponding transfer functions are given by delta distributions.

The Monte Carlo events have been produced with an input top-quark mass at $160 \mathrm{GeV}$. They have been required to contain the right number of jets and muons in the final state, but no kinematics cuts have been applied. A sample of $N=20$ events is passed to MadWeight for the evaluation of the weights. The mass of the top quark is extracted through the maximization of the likelihood, or equivalently the minimization of $-\ln \left(L\left(m_{t}\right)\right)$.

$$
-\ln (L)=-\sum_{i=1}^{N} \ln \left(P\left(x_{i} ; m_{t}\right)\right)+N * A c c\left(m_{t}\right),
$$

where $\operatorname{Acc}\left(m_{t}\right)$ correct the potential bias introduced both by the acceptance of the detector and by the cuts imposed on the sample (this correction term is computed by standard Monte-Carlo techniques).

The values of $-\ln \left(L\left(m_{t}\right)\right)$ for different assumptions of $m_{t}$ are displayed in Fig. 1. A fit by a parabolic distribution provides the best estimation of $m_{t}$ as well as the associated statistical error: $m_{t}=158.9 \pm 2.3_{\text {stat }} \mathrm{GeV}$ which is compatible with the generated mass.

\subsection{Charged Higgs studies in top-quark decays}

The presence of charged Higgs is predicted by various models like 2HDM or MSSM. A charged scalar doesn't appear in the standard model, so that its observation would be a clear 


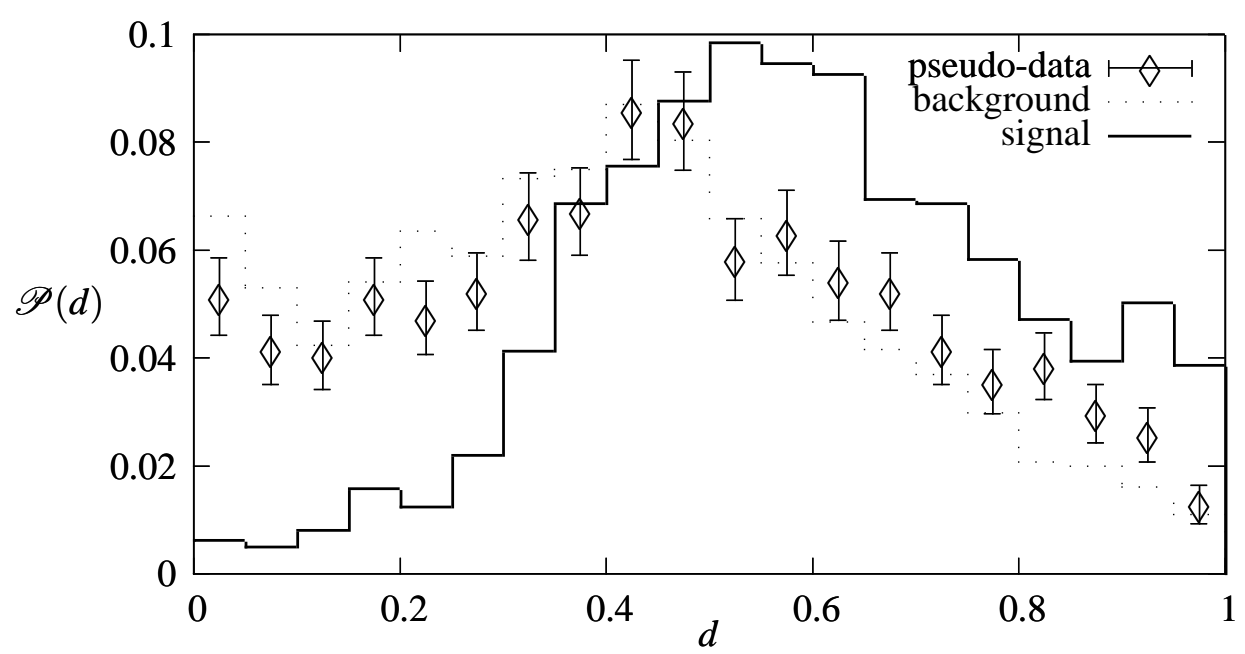

Figure 2: Distribution of events with respect to the discriminant $d$ for a sample of pure events (solid line), of background events (dotted line) and of mixed events (points).

signal of new physics. In this example, we consider the production of a light charged Higgs $\left(m_{H^{+}}=100 \mathrm{GeV}\right)$ originating from the decay of a top quark. We restrict ourself to the following topology:

$$
p p \rightarrow\left(t \rightarrow b\left(H^{+} \rightarrow \mu^{+} v_{\mu}\right)\right)\left(\bar{t} \rightarrow \bar{b}\left(W^{-} \rightarrow \mu^{-} \bar{v}_{\mu}\right)\right)
$$

Note that if we turn the charged Higgs in a $W$ boson, the above process reduces to the Standard Model top-quark pair production in the di-leptonic channel. This irreducible background cannot be removed by a simple cut analysis. For the purpose of simplification, we will consider only this background in the following.

The problem of identifying a charged-Higgs signal in top-quark pair events can be expressed in the framework of the matrix element method. Using the weights defined in Eq. 2.1, we build an event-by-event discriminating variable $\mathrm{d}(\mathrm{x})[8]$ :

$$
d(x)=\frac{P_{S}(x)}{P_{S}(x)+P_{B}(x)}
$$

where $P_{S}(x)$ and $P_{B}(x)$ are the weights corresponding respectively to the signal and the background hyppotheses.

The distribution of events with respect to this variable $d$ is plotted in Fig. 2. The solid (resp. dotted) line represents the normalized distribution for a sample of pure signal (resp. background) events. As the two types of sample lead to different shapes in the discriminant variable $d$, this allows us to extract experimentally the fraction of signal events in a mixed sample. In our pseudo experimental sample, we consider 1011 events with respectively $25.9 \%$ and $74.1 \%$ of signal/background events. The related distributions are, compared to the background curve $\left[\mathscr{P}_{B}(d)\right]$, systematically shifted towards the signal one $\left[\mathscr{P}_{S}(d)\right]$. This is interpreted as the presence of signal events. Their relative number can be estimated by fitting the experimental points with the curve $(1-r) \mathscr{P}_{B}(d)+r \mathscr{P}_{S}(d)$ where $r$ represents the fraction of signal events in the sample. The best fit is obtained for $r=21 \pm 4 \%$, compatible to expected value, which shows that the matrix element method succeeds to identify a charged Higgs in top-quark pair events under the above simplifying assumptions. 


\section{Conclusion}

We have presented a new algorithm to automatically compute the weights needed in the matrix element method. Given an arbitrary process, our code produces a phase-space generator optimized to integrate the product of the matrix element and the resolution function.

This tool gives a practical access to a large number of possible applications. Indeed, the matrix element method offers a powerful prescription to discriminate between different theoretical hypotheses. This approach can be used not only for the measurement of Standard Model parameters but also for the observation of new physics. For the purpose of studying the new horizons opened by this method, MadWeight provides the resource for the practical evaluation of the weights, sparing the user to focus on the integration techniques.

\section{Acknowledgement}

We would like to express our gratitude to V. Lemaître and F. Maltoni for their help and advice. P. Artoisenet is a Research Fellow of the Fonds National de la Recherche Scientifique, Belgium. This work is supported by the Institut Interuniversitaire des Sciences Nucléaires, and by the Belgian Federal Office for Scientific, Technical and Cultural Affairs through the Interuniversity Attraction poles (IAP) P6/11.

\section{References}

[1] A. Abulencia et al. [CDF Collaboration], Phys. Rev. D 75 (2007) 031105 [arXiv:hep-ex/0612060].

[2] A. Abulencia et al. [CDF Collaboration], Phys. Rev. Lett. 99 (2007) 182002 [arXiv:hep-ex/0703045].

[3] V. M. Abazov et al. [D0 Collaboration], Phys. Rev. D 74 (2006) 092005 [arXiv:hep-ex/0609053].

[4] V. M. Abazov et al. [D0 Collaboration], Phys. Rev. D 75 (2007) 092001 [arXiv:hep-ex/0702018].

[5] K. Kondo, J. Phys. Soc. Jap. 57 (1988) 4126.

[6] R. H. Dalitz and G. R. Goldstein, Phys. Rev. D 45 (1992) 1531.

[7] A. Abulencia et al. [CDF Collaboration], CDF note 9500 (2008).

[8] V. M. Abazov et al. [D0 Collaboration], Phys. Rev. D 78 (2008) 012005 [arXiv:hep-ex/0803.0739].

[9] K. Kondo, J. Phys. Conf. Ser. 53 (2006) 202.

[10] M. F. Canelli. Ph.D. Thesis University of Rochester (2003).

[11] G. P. Lepage, Journal of Computational Physics 27 (1978) 192.

[12] S. Weinzierl, [arXiv:hep-ph/0006269].

[13] P. Artoisenet, V. Lemaître, F. Maltoni, O. Mattelaer, in progress.

[14] F. Maltoni and T. Stelzer, JHEP 0302 (2003) 027 [arXiv:hep-ph/0208156].

[15] T. Sjostrand, S. Mrenna and P. Skands, JHEP 0605 (2006) 026 [arXiv:hep-ph/0603175].

[16] J. Conway, http://www.physics.ucdavis.edu/conway/research/software/pgs/pgs4-general.htm.

[17] P. A. Movilla Fernandez [CDF Collaboration and D0 Collaboration], Frascati Phys. Ser. 44 (2007) 259 [arXiv:hep-ex/0705.3910]. 